\title{
EFEITO DE DIFERENTES ÉPOCAS DE DESFOLHA E DE COLHEITA NA COMPOSIÇÃO DO VINHO CABERNET SAUVIGNON ${ }^{1}$
}

\author{
EFFECT OF DIFFERENT DEFOLIATION AND HARVESTING TIMES \\ IN THE COMPOSITION OF CABERNET SAUVIGNON WINE
}

\author{
Vitor Manfroi $^{2}$ Alberto Miele ${ }^{3}$ Luiz Antenor Rizzon ${ }^{3}$ \\ Carlos Iguassu Nogueira Barradas ${ }^{4}$ Luciano Manfroi $^{5}$
}

RESUMO

O trabalho foi realizado em Santana do Livramento. Rio Grande do Sul, com o objetivo de avaliar o efeito de épocas de desfolha e de colheita sobre a composição físico-química do vinho Cabernet Sauvignon. O experimento foi conduzido num fatoriat incompleto, com quatro épocas de desfolha e três épocas de colheita, realizadas em função de uma data ideal de colheita, totalizando onze tratamentos, com três repetições. A intensidade de desfolha foi de aproximadamente 25\%. Os vinhos foram elaborados através de microvinificações. Os resultados mostraram que a época de desfolha não afetou de forma significativa a maioria das variáveis analisadas no vinho, à exceçao do álcool, açúcares redutores e extraio seco. A época de colheita, por outro lado, influiu significativamente na maior parte das variáveis, exceto na acidez total e na acidez volátil, o que sugere uma maior influência deste fator sobre a composição $e$ qualidade do vinho

Palavras-chave: composição físico-química, vinificação.

\section{SUMMARY}

The fleld work was carried out in Santana do Livramento, State of Rio Grande do Sul, Brazil, aiming to evaluate the effect of defotiation and harvesting times on the physicchemical composition of Cabernet Sauvignon wines. The experimental design was an incompletely factorial with four defotiation times and three harvesting limes at on ideal harvest date with a total of eleven treatments in three replicales. The defotiation intensity was about $25 \%$ and wines were elaborated in small volumes The results showed that the time of defotiation did not have significam effect on the majority of the variables anatyzed, exception on the alcohot, reducing sugars and dry extract. On the other hand, the harvesting time had a significant effect on almost ali variables, exception to the total and votatile acidities. These results suggests a greater influence of the harvesting time on the composition and quatity of Cabernet Sauvignon wine.

Key words: physic-chemical composition, vinification.

\section{INTRODUÇÃO}

A desfolha tem o objetivo de minimizar problemas climáticos encontrados durante a maturação da uva, como precipitação e umidade relativa do ar elevadas. O manejo do dossel vegetativo permite uma maior insolação e aeração do vinhedo, favorecendo o microclima próximo às folhas e aos frutos, e ao aumento da qualidade da uva.

\footnotetext{
${ }^{1}$ Parte da Dissertação de Mestrado em Fitotecnia apresentada pelo primeiro autor.

${ }^{2}$ Engenheiro Agrônomo, MsC., Departamento de Tecnologia dos Alimentos, Instituto de Ciência e Tecnologia dos Alimentos, Universidade Federal do Rio Grande do Sul (UFRGS), Av.: Bento Gonçalves, 9500, 90540-000, Porto Alegre - RS. Autor para correspondência.

${ }^{3}$ Engenheiro Agrônomo, PhD., EMBRAPA, Centro Nacional de Pesquisa de Uva e Vinho.

${ }^{4}$ Engenheiro Agrônomo, PhD., Departamento de Horticultura e Silvicultura, Faculdade de Agronomia, UFRGS.

${ }^{5}$ Engenheiro Agrônomo, Mestrando em Fitotecnia, Departamento de Horticultura e Silvicultura, Faculdade de Agronomia, UFRGS.
} 
Experimentos conduzidos em vários países, como França, Itália, Estados Unidos, África do Sul, Austrália, Nova Zelândia e Chile, evidenciam que o manejo do microclima da copa é um meio efetivo de aumentar a qualidade do vinho.

No que se refere à composição analítica dos vinhos, poucas variáveis analisadas mostraram diferenças significativas (PSZCZÓLKOWSKI $\boldsymbol{e t}$ al., 1985; SMART, 1985; REYNOLDS et al., 1986; FREESE, 1988; ILAND, 1988). No entanto, análises sensoriais demonstraram que vinhos elaborados de uvas em condições de microclima manejado obtiveram escores superiores, e foram considerados de melhor qualidade (CARBONNEAU, 1978; SMART, 1984; PSZCZÓLKOWSKI et al., 1985; HUNTER \& VISSER, 1991).

Modificações aromáticas e na composição, principalmente, dos constituintes da película, resultantes da prática da desfolha, poderiam ser um instrumento do enólogo para obtenção de vinhos com tipificação e características próprias. Portanto, o objetivo do trabalho foi estudar o efeito de épocas de desfolha e de colheita sobre a composição físico-química do vinho Cabemet Sauvignon.

\section{MATERIAIS E MÉTODOS}

O experimento foi conduzido durante o ciclo vegetativo 1990/91, em vinhedo no município de Santana do Livramento, RS, Brasil, situado a $30^{\circ} 48^{\prime} \mathrm{S}$ e $55^{\circ} 22^{\prime} \mathrm{W}$, em uma altitude de $210 \mathrm{~m}$. O clima da região é classificado como subtropical úmido, tipo fundamental $\mathrm{Cfa}$, segundo classificação de Kõeppen (MORENO, 1961), com temperatura média anual de $18,2^{\circ} \mathrm{C}$, e precipitação em tomo de $1.400 \mathrm{~mm}$ anuais. Pelo levantamento de Reconhecimento dos Solos do Estado do Rio Grande do Sul (BRASIL, 1973), os solos da região são classificados como Bruno Gleizado Distrófico, apresentando baixos teores de matéria orgânica, friáveis em todo o perfil, com moderada drenagem e baixa incidência de afloramentos e perdas.

$\mathrm{O}$ vinhedo de Cabemet Sauvignon onde foram instalados os experimentos era conduzido em espaldeira, com espaçamento de $3,5 \mathrm{~m}$ entre fileiras e $2,0 \mathrm{~m}$ entre plantas. As plantas tinham dez anos de idade, e foram enxertadas em 1980 sobre o portaenxerto S04. A poda adotada foi a de cordão esporonado.

A desfolha nas videiras foi realizada na base dos ramos, retirando-se as folhas até uma altura de 20-30cm, deixando-se os cachos expostos ao sol. A intensidade da desfolha foi de aproximadamente $25 \%$ do número de folhas.
O experimento foi conduzido no delineamento completamente casualizado, num fatorial incompleto, com quatro épocas de desfolha e três épocas de colheita, feitas a partir do inicio da maturação. As desfolhas e as colheitas foram realizadas em função de uma data ideal de colheita (DÍC), que foi estimada a partir de registros fenológicos do vinhedo em anos anteriores, e em função da evolução da maturação naquela safra.

Foram efetuados 11 tratamentos, com três repetições/tratamento, cada parcela com dez plantas. As datas das desfolhas e colheitas constam da Figura 1.

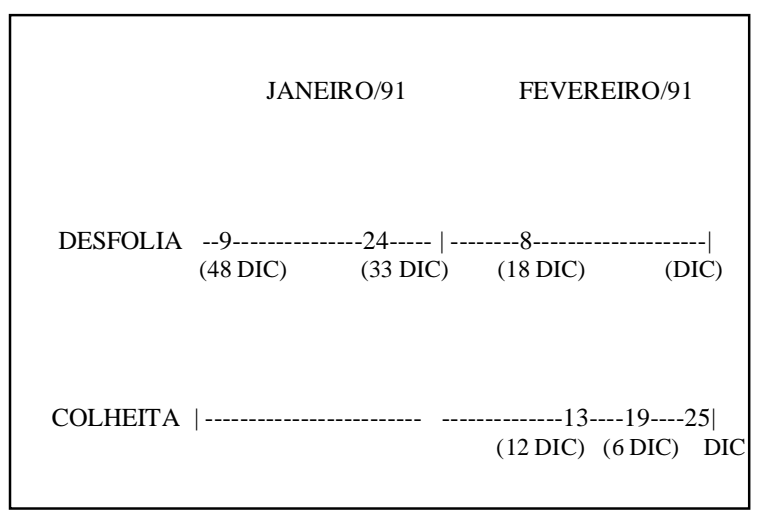

Figura 1 - Datas das épocas de desfolha e de colheita, em função das datas ideais de colheita (DIC), realizadas em "Cabernet Sauvignon". Santana do Livramento - RS. Clico vegetativo 1990/1991.

Nas datas previstas de colheita, foram amostrados 30 cachos/planta, totalizando cerca de 30 $\mathrm{Kg} /$ parcela. As uvas assim colhidas foram acondicionadas em caixas de plástico de $20 \mathrm{Kg}$, e transportadas até o Centro Nacional de Pesquisa de Uva e Vinho (EMBRAPA-CNPUV), em Bento Gonçalves, para serem vinificadas.

Ao chegarem ao CNPUV, as uvas foram pesadas, desengaçadas e esmagadas. Cerca de 17 de mosto foram colocados em garrafões de vidro de 20', aos quais acrescentaram-se $\mathrm{SO}_{2}(50 \mathrm{mg} / \mathrm{Kg}$ de uva) e leveduras liofolizadas $(200 \mathrm{mg} / \mathrm{Kg}$ uva de um complexo Saccharomyces cerevisae e Saccharomyces bayanus). Os garrafões foram tampados com válvulas de Müller e colocados numa sala com temperatura de aproximadamente 22 "C.

Após cinco dias de maceração, fez-se a descuba, permitindo que o restante da fermentação se efetuasse apenas com o liquido. Depois de quinze dias, fez-se uma trasfega, separando-se a borra, e colocaram-se os vinhos em garrafões de 5L. 
Quatro meses após a trasfega, quando todos os vinhos haviam realizado a fermentação malolática, verificada por cromatografía de papel, estabilizaram-se os vinhos pelo frio $\left(-4^{\circ} \mathrm{C}\right)$ por um período de sete dias, filtrando-os com um filtro de placas de celulose.-Após, envasaram-se seis garrafas/parcela, as quais foram mantidas em sala com temperatura a $20^{\circ} \mathrm{C}$, dispostas horizontalmente sobre estrados de madeira. O vinho permaneceu assim até o início das análises químicas, realizadas entre novembro/91 aabril/92.

A metodologia empregada na maioria das análises físico-químicas foram propostas por AMERINE \& OUGH (1974), RIBÉREAU-GAYON et al (1975). Os ácidos orgânicos foram determinados por cromatografía líquida de alta performance (HPLC), segundo AUGUSTE (1979). Os dados obtidos foram submetidos à análise de variância e ao teste de Tukey em nível de 5\% de probabilidade.

\section{RESULTADOS E DISCUSSÃO}

Quanto às características físico-químicas do vinho, encontraram-se algumas diferenças entre épocas de desfolha. No entanto, não na magnitude apresentada pela época de colheita, cuja influência foi muito marcante.

As variáveis analisadas constam da Tabela 1. O teor de álcool aumentou significativamente ao se aproximar a época ideal da colheita, para os tratamentos com desfolha. O tratamento controle (sem desfolha) só teve teores de álcool mais elevados na colheita na DIC, indicando um incremento maior desta variável nos tratamentos com desfolha. Apesar de não se detectarem diferenças marcantes na composição dos frutos (MANFROI, 1993), pode-se verificar que os tratamentos com desfolha propiciaram condições que permitiram a elaboração de vinhos com maior graduação alcoólica, a exemplo do que encontraram REYNOLDS $\boldsymbol{e t}$ al. (1986) e SMART et al. (1990) em vinhos elaborados a partir de uvas expostas ao sol.

A densidade dos vinhos não foi influenciada pelas épocas de desfolha, e somente aqueles elaborados com uvas colhidas na DIC obtiveram valores superiores àqueles elaborados com uvas colhidas 12 dias antes da DIC.

$\mathrm{O}$ pH foi influenciado pela época de colheita, sendo que a diferença entre os efeitos de épocas de colheita foi mais marcante nos tratamentos em que as desfolhas foram realizadas a 48 e 33 dias da DIC. Por outro lado, a acidez total praticamente não diferiu entre épocas de desfolha e de colheita.

\begin{tabular}{|c|c|c|c|}
\hline \multirow[b]{2}{*}{ EPOCA DESFOLHA } & \multicolumn{3}{|c|}{ ÉPOCA COLHEITA ${ }^{2}$} \\
\hline & C $22 \mathrm{DIC}$ & $\mathrm{C} 6 \mathrm{DIC}$ & $\mathrm{C} \mathrm{DlC}$ \\
\hline $\begin{array}{l}\text { D48 DIC } \\
\text { D33 DIC } \\
\text { D18 DIC } \\
\text { SEM DESFOLHA }\end{array}$ & $\begin{array}{l}11,84 \text { a } \\
1156 \text { a } \\
1159 \text { a B }\end{array}$ & $\begin{array}{l}\text { Alcool ( } \mathrm{GL}) \\
12,60 \mathrm{a} \mathrm{B} \\
12,68 \mathrm{aB} \\
12,33 \mathrm{ab} \mathrm{B} \\
11,84 \mathrm{~b} \mathrm{~B}\end{array}$ & $\begin{array}{l}14,32 \mathrm{aA} \\
14,47 \mathrm{aA} \\
14,41 \mathrm{aA} \\
12,83 \mathrm{bA}\end{array}$ \\
\hline $\begin{array}{l}\text { D48 DIC } \\
\text { D33 DIC } \\
\text { D18 DIC } \\
\text { SEM DESFOLHA }\end{array}$ & $\begin{array}{l}0,9941 \mathrm{a} \mathrm{B} \\
0,9943 \mathrm{a} \mathrm{B} \\
0,9941 \mathrm{a} \mathrm{B}\end{array}$ & $\begin{array}{c}\text { Densidade } \\
0,9942 \mathrm{a} \mathrm{B} \\
0,9949 \mathrm{a} \mathrm{AB} \\
0,9945 \mathrm{a} A \\
0,9949 \mathrm{a} \mathrm{AB}\end{array}$ & $\begin{array}{r}0,9957 \mathrm{aA} \\
8 \quad 0,9955 \mathrm{aA} \\
0,9951 \mathrm{aA} \\
\mathrm{B} 0,9960 \mathrm{aA}\end{array}$ \\
\hline $\begin{array}{l}\text { D48 DIC } \\
\text { D33 DIC } \\
\text { D18 DIC } \\
\text { SEM DESFOLHA }\end{array}$ & $\begin{array}{l}3,92 \mathrm{aC} \\
3,89 \mathrm{aC} \\
3,88 \mathrm{a} \mathrm{B}\end{array}$ & $\begin{array}{l}\mathrm{pH} \\
4,08 \mathrm{a} \mathrm{B} \\
4,11 \mathrm{a} \mathrm{B} \\
4,04 \mathrm{a} \mathrm{B} \\
4,04 \mathrm{a} \mathrm{B}\end{array}$ & $\begin{array}{l}4,29 \text { a } A \\
4,27 \text { a } A \\
4,21 \text { a } A \\
4,17 \text { a } A\end{array}$ \\
\hline $\begin{array}{l}\text { D48 DIC } \\
\text { D33 DIC } \\
\text { DI8 DIC } \\
\text { SEM DESFOLHA }\end{array}$ & $\begin{array}{l}63 \mathrm{aA} \\
70 \mathrm{aA} \\
67 \mathrm{aA}\end{array}$ & $\begin{array}{l}\text { total (meq/) } \\
58 \mathrm{a} \mathrm{B} \\
59 \mathrm{a} \mathrm{B} \\
60 \mathrm{aA} \\
57 \mathrm{a} \mathrm{A}\end{array}$ & $\begin{array}{l}59 \text { a A } \\
61 \text { a A } \\
62 \text { a A } \\
61 \text { a A }\end{array}$ \\
\hline $\begin{array}{l}\text { D48 DIC } \\
\text { D33 DIC } \\
\text { D } 18 \text { DIC } \\
\text { SEM DESFOLHA }\end{array}$ & $\begin{array}{l}\text { Acidez } \\
9 \mathrm{aA} \\
1 \mathrm{aA} \\
10 \mathrm{aA}\end{array}$ & $\begin{array}{l}\text { volátil (meq/l) } \\
9 \mathrm{aA} \\
10 \mathrm{aA} \\
8 \mathrm{aA} \\
7 \mathrm{aA}\end{array}$ & $\begin{array}{l}10 \mathrm{aA} \\
10 \mathrm{aA} \\
10 \mathrm{aA} \\
9 \mathrm{aA}\end{array}$ \\
\hline $\begin{array}{l}\text { D48 DIC } \\
\text { D33 DIC } \\
\text { D18 DIC } \\
\text { SEM DESFOLHA }\end{array}$ & $\begin{array}{c}\text { Acido } \\
1,45 \mathrm{aB} \\
150 \mathrm{aA} \\
1,59 \mathrm{aA}\end{array}$ & $\begin{array}{c}\text { Tartarico (a/l) } \\
138 \mathrm{a} \mathrm{B} \\
157 \mathrm{aA} \\
1.41 \text { a A } \\
155 \mathrm{aA}\end{array}$ & $\begin{array}{l}1,83 \mathrm{aA} \\
1,87 \mathrm{aA} \\
1,63 \mathrm{aA} \\
1.69 \mathrm{aA}\end{array}$ \\
\hline $\begin{array}{l}\text { D48 DIC } \\
\text { D33 DIC } \\
\text { D18 DIC } \\
\text { SEM DESFOLHA }\end{array}$ & $\begin{array}{l}\text { Acido } \\
1,13 \text { a B } \\
1,27 \text { a B } \\
1,18 \text { a B }\end{array}$ & $\begin{array}{c}\text { Málico }(\mathrm{g} / \mathrm{l}) \\
1,36 \mathrm{aB} \\
1,64 \mathrm{a} \mathrm{AB} \\
1,41 \mathrm{aA} \\
1,41 \mathrm{aAB}\end{array}$ & $\begin{array}{l}1,94 \mathrm{aA} \\
1,89 \mathrm{aA} \\
1,68 \mathrm{aA} \\
1,70 \mathrm{aA}\end{array}$ \\
\hline $\begin{array}{l}\text { D48 DIC } \\
\text { D33 DIC } \\
\text { DI8 DIC } \\
\text { SEM DESFOLHA }\end{array}$ & $\begin{array}{l}\text { Alcalnidade } \\
32,1 \mathrm{a} \mathrm{C} \\
33,6 \mathrm{a} \mathrm{B} \\
31,9 \mathrm{aB}\end{array}$ & $\begin{array}{l}35 \text { cinzas (meq } \\
35,9 \text { a B } \\
37,7 \text { a A } \\
35,2 \text { a A } \\
36,1 \text { a A }\end{array}$ & $\begin{array}{l}40,0 \mathrm{a} \mathrm{A} \\
36,8 \mathrm{aAB} \\
37,6 \mathrm{aAA} \\
38,9 \mathrm{aA}\end{array}$ \\
\hline $\begin{array}{l}\text { D48 DIC } \\
\text { D33 DIC } \\
\text { DI8 DIC } \\
\text { SEM DESFOLHA }\end{array}$ & $\begin{array}{l}\text { Acúcares } \\
1,38 \mathrm{aB} \\
1,27 \mathrm{aC} \\
1,30 \mathrm{a} \mathrm{B}\end{array}$ & $\begin{array}{l}\text { edutores }(\mathrm{g} / \mathrm{f}) \\
1,75 \mathrm{a} \mathrm{B} \\
1,82 \mathrm{a} \mathrm{B} \\
1,78 \mathrm{aB} \\
1,65 \mathrm{a} \mathrm{AB}\end{array}$ & $\begin{array}{l}2,66 \mathrm{ab} A \\
2,75 \mathrm{aA} \\
2,47 \mathrm{abA} \\
2,09 \mathrm{bA}\end{array}$ \\
\hline $\begin{array}{l}\text { D48 DIC } \\
\text { D33 DIC } \\
\text { DI8 DIC } \\
\text { SEM DESFOLHA }\end{array}$ & $\begin{array}{l}24,9 \mathrm{aB} \\
23,5 \mathrm{aC} \\
23,2 \mathrm{aC}\end{array}$ & $\begin{array}{l}\operatorname{seco}(\mathrm{g} / \mathrm{A} \\
28,3 \mathrm{a} \mathrm{B} \\
29,7 \mathrm{a} \text { B } \\
27,3 \mathrm{a} \mathrm{B} \\
26,9 \mathrm{a} \mathrm{B}\end{array}$ & $\begin{array}{l}37,6 \mathrm{aA} \\
37,6 \mathrm{aA} \\
36,6 \mathrm{abA} \\
33,3 \mathrm{bA}\end{array}$ \\
\hline $\begin{array}{l}\text { D48 DIC } \\
\text { D33 DIC } \\
\text { D18 DIC } \\
\text { SEM DESFOLHA }\end{array}$ & $\begin{array}{l}3,90 \mathrm{a} \mathrm{B} \\
3,87 \mathrm{aB} \\
3,63 \mathrm{aB}\end{array}$ & $\begin{array}{l}1(g / 1) \\
4,20 \mathrm{aB} \\
4,42 \mathrm{a} \mathrm{AB} \\
4,0 \mathrm{aB} \\
4,00 \mathrm{aB}\end{array}$ & $\begin{array}{l}5,22 \mathrm{a} \mathrm{A} \\
4,95 \mathrm{aAA} \\
4,80 \mathrm{aA} \\
4,75 \mathrm{a} \mathrm{A}\end{array}$ \\
\hline
\end{tabular}

I Medias seguidas por letras minúsculas distintas, na coluna, e po maiúsculas, na linha idferem entre si pelo teste de Tukey em nivel de $5 \%$ de probabilidade

$2 \mathrm{Cl} 2 \mathrm{DIC}$-Cotheita 12 dias antes da data udeal de colheita; C6 DIC-Colheita 6 dias antes da data ideal de colheita: C DIC-Colheita na data ideal de colleita.

3 D48DIC - Desfolha 48 dias antes da data ideal de colheita D33DIC-Desfolha 33 dias antes da data ideal de colheita: DI8DIC - Desfolha 18 dias antes da data ideal de colheita 
SMART (1984) e REYNOLDS \& WARDLE (1989) também não encontraram diferenças na acidez total de vinhos obtidos de cachos submetidos a diferentes níveis de sombreamento.

A acidez volátil indica que, mais do que as práticas de manejo das plantas, a tecnologia empregada na elaboração dos vinhos foi adequada. PSZCZÓLKOWSKI et al. (1985) informaram que vinhos obtidos de cachos expostos ao sol tiveram maior acidez volátil, devido a uma fermentação mais lenta e prolongada.

Os ácidos tartárico e málico dos vinhos não foram afetados pela desfolha. O ácido tartárico, pela sua própria estrutura e relação com seus sais, explica em grande parte a não ocorrência de diferenças para épocas de colheita. Devido ao tratamento para estabilização dos vinhos, feito pela refrigeração, ocorreu a precipitação de sais de ácido tartárico, o que propiciou vinhos com teores similares deste ácido.

Para o ácido málico houve diferenças significativas entre épocas de colheita, com maiores valores nos vinhos da colheita na DIC, ainda que, na prática, tais diferenças não representem muito. Nos vinhos tintos brasileiros, em geral, deseja-se que ocorra a fermentação malolática, e, foi o que se verificou com todos os vinhos experimentais. Devido a fatores de ordem diversa, dos quais o $\mathrm{pH}$ talvez tenha sido o mais importante, os vinhos elaborados nas primeiras colheitas tiveram uma fermentação malolática mais intensa, o que levou aquele grupo de vinhos aos menores teores de ácido málico.

A alcalinidade das cinzas praticamente não foi afetada pela época de desfolha. No entanto, houve um acréscimo significativo da mesma em função da época de colheita. Isto pode ser atribuído, em boa parte, a maior salifícação dos ácidos orgânicos, principalmente do ácido tartárico, pela combinação com o $\mathrm{K}$ e o $\mathrm{Ca}$. A desfolha aos 48 dias da DIC mostrou diferenças significativas entre as três épocas de colheita. Possivelmente, a desfolha mais antecipada permitiu uma salifícação mais intensa dos ácidos, a exemplo dos resultados obtidos por CRIPPEN Jr. \& MORRISON (1986), que encontraram maiores concentrações de tartaratos e malatos em mostos de uvas expostas ao sol.

Os açúcares redutores residuais dos vinhos foram significativamente afetados pela época de colheita: foram superiores na DIC, e nesta mesma época, o tratamento sem desfolha apresentou o menor valor, ainda que superior a $2 \mathrm{~g} / \mathrm{L}$ e que na prática pouco representa, já que todos os vinhos são considerados secos. Os maiores valores de açúcares redutores nos vinhos da colheita na DIC podem ser explicados pelo maior teor inicial dos mostos e, conseqüientemente, pelo teor alcoólico elevado destes vinhos. Isto, certamente, não permitiu que as leveduras metabolizassem por completo a glicose e a frutose.

$\mathrm{O}$ extrato seco, que mede a quantidade de substâncias fixas presentes no vinho, foi significativamente afetado pela época de colheita. Os níveis mais elevados foram encontrados na colheita na DIC, comprovando aumento da maioria dos componentes à medida que ocorreu o retardamento da colheita. Da mesma maneira, na colheita na DIC o tratamento sem desfolha propiciou o menor valor de extrato seco. SMART et al. (1990) também encontraram maior extrato seco em vinhos de plantas dispostas de maneira a captar maior insolação nos seus cachos.

As cinzas não foram afetadas pela época de desfolha. Todavia, seus teores aumentaram, sendo significativamente superiores na colheita na DIC. Este comportamento reflete um incremento de minerais e outras substâncias constituintes da parte inorgânica dos vinhos, extraídas com maior intensidade nas uvas maduras.

\section{CONCLUSÕES}

A época de desfolha não tem efeito significativo sobre a maior parte das variáveis avaliadas: as mais afetadas pela época de desfolha são o álcool e o extrato seco e,

a época de colheita afeta de maneira mais intensa a composição físico-quimica dos vinhos do que a época da desfolha, denotando uma maior influência do momento da colheita dos frutos sobre a qualidade final do vinho: somente a acidez total e acidez volátil não são por ela significativamente influenciadas.

\section{REFERÊNCIAS BIBLIOGRÁFICAS}

AMERINE, M. A.. OUGH. C. S. Analisis de vinos y mostos. Zaragoza: Acribia, 1974. 158p.

AUGUSTE, M. H. Appiication de Ia chromatographie en phase liquide à haute pression à 1 'analyse dês moüts dês vins. Bordeaux: Institute d'0enologie, 1979. 135 p. (Tese de Doutorado).

BRASIL. Ministério da Agricultura. Departamento Nacional de Pesquisa Agropecuária. Divisão de Pesquisa Pedológica. Levantamento de reconhecimento dos solos do Estado do Rio Grande do Sul. Recife: Ministério da Agricultura. 1973. 431 p. (Boletim Técnico. 30)

CARBoneAu, A. Appiieations de l'étude de Ia photosynthèse sur différents systèmes de conduite à Ia seléetion de varietés de vigne. Bordeaux, 1977. In: SYMPOS1UM 1NTERNATIONAL 
AMÉLIORATION DE LA VIGNE, 2., 1978. Bordeaux. Genétique et Amélioration de Ia Vigne Paris, INRA, 1978. p.313-20.

CRIPPEN Jr., D. D., MORRISON, J. C. The effects of sun exposure on the compositional development of Cabemet Sauvignon berries. Am J Enol Vitic, Davis, v. 37, n. 4, p. 235-42.1986.

FREESE, P. K. Canopy modification and fruit composition, Auckland, 1988. In: INTERNATIONAL SYMPOSIUM FOR COOL CLIMATE VITICULTURE AND OENOLOGIE, 2. 1988, Auckland. Proceedings... Auckland, New Zealand Soe. Vitie. Oenol, 1988. p. 134-6.

HUNTER, J. J., V1SSER, J. H. The effect ofpartial defoliation on quality characteristics of Vitis vinifera L. cv. Cabemet Sauvignon grapes. II. Skin color, skin sugar, and wine quality. Am J Enol Vitic, Davis, v. 42, n. 1, p. 13-8. 1991.

ILAND, P. G. Leafremoval effects on fruit composition, Auckland, 1988. In: INTERNATIONAL SYMPOSIUM FOR COOL CLIMATE VITICULTURE AND OENOLOGIE, 2. 1988, Auckland. Proceedings... Auckland, New Zealand Soe. Vitic. Oenol, 1988. p. 137-8.

MANFR01, V. Efeito de épocas de desfolha e de colheita sobre a maturação e qualidade da uva e do vinho 'Cabemet Sauvignon'. Porto Alegre, RS. 152 p. Dissertação (Mestrado em Fitotecnia) - Curso de Pós-graduação em Fitotecnia. Universidade Federal do Rio Grande do Sul, 1993.

MOREnO, J. A. Clima do Rio Grande do Sul. Porto Alegre:
Secretaria da Agricultura do, Rio Grande do Sul, 1961. 42 p.

PSZCZÓLKOWSKI, Ph., MORALES, A., CAVA, S. Composition química y calidad de mostos y vinos obtenidos de racimos diferentemente asoleados. Cienc Invest Agr, Santiago, v. 12, n. 3, p. 181-8. 1985.

REYNOLDS, A. G., POOL, R. M., MATTICK, L. R. Influence of cluster exposure on fruit composition and wine quality of Seyval blanc grapes. Vitis, Landau, v. 25, p. 85-95. 1986.

REYNOLDS, A. G.. WARDLE. D. A. Impact of various canopy manipulation techniques on growth, yield, fruit composition, and wine quality ofGewurztraminer. Am J Enol Vitic, Davis, v.40,n.2,p.121-9. 1989.

RIBÉREAU-GAYON, J., PEYNAUD, E., RIBÉREAU-GAYON, P., et al. Sciences et techniques du vin. Paris: Bordas, 1975. T. $1.643 \mathrm{p}$.

SMART, R. E. Canopy microclimates and effects on wine quality, Perth, 1983. In: AUSTRALIAN WINE RESEARCH INSTITUTCONFERENCE, 1984, Perth. Proceeding... Perth, 1984.p.113-32.

SMART, R. E. Principies of grapevine canopy microclimate manipulation with implications for yield and quality - A review. Am J Enol Vitic, Davis, v. 36. n. 3, p. 230-9. 1985.

SMART. R. E., D1CK J. K., GRAVETT. I. M.. et al. Canopy management to improve grape, yield and wine quality Principies and practices. S Afr J Enol Vitic. Stellembosch. v. 11, n. 1, p. 3-17. 1990.

Ciência Rural, v. 27n. 11997 